Article

\title{
Simple Particle Model for Low-Density Granular Flow Interacting with Ambient Fluid
}

\author{
Hirofumi Niiya ${ }^{1, *(\mathbb{D})}$, Akinori Awazu ${ }^{2}$ and Hiraku Nishimori ${ }^{2}$ \\ 1 Center for Transdisciplinary Research, Niigata University, 8050 Ikarashi-nino-cho, Nishi-ku, \\ Niigata 950-2181, Japan \\ 2 Department of Mathematical and Life Sciences, Hiroshima University, 1-3-1, Kagamiyama, \\ Higashi-Hiroshima 739-8526, Japan; awa@hiroshima-u.ac.jp (A.A.); nishimor@hiroshima-u.ac.jp (H.N.) \\ * Correspondence: niiya@gs.niigata-u.ac.jp
}

Received: 13 January 2020; Accepted: 8 February 2020; Published: 13 February 2020

\begin{abstract}
To understand the time evolutions of frontal speed and shape in a low-density granular flow, we propose a simple particle model. This model solves the equation of motion for each particle and simulates the time evolution of low-density granular flow. Spherical particles constituting a low-density granular flow slide on a slope at a steeper angle than the angle of repose. The particle motion is determined based on three forces: gravity as the driving force, repulsive force due to particle collision, and drag force due to the particle interaction through the ambient fluid. Two-dimensional numerical simulations of this model are conducted on the slope: the $x-y$ plane parallel to the slope and the $x-z$ plane perpendicular to the slope. In the $x-y$ plane, particles aggregate at the moving front of the granular flow, and subsequently, flow instability occurs as a wavy pattern. This flow pattern is caused by the interparticle interaction arising from the drag force. Additionally, a vortex convection of particles is formed inside the aggregations. Simultaneously, particle aggregation is also found at the moving front of the granular flow in the $x-z$ plane. The aggregation resembles a head-tail structure, where the frontal angle against the slope approaches $60^{\circ}$ from a larger angle as time progresses. Comparing the numerical result by varying the particle size reveals that the qualitative dynamics of the granular flow are independent of particle size. Although the model is not realistic, our study presents a new particle-based approach that elucidates the dynamics of low-density granular flow.
\end{abstract}

Keywords: granular avalanches; mathematical modeling; particle model; numerical simulation; pattern formation

\section{Introduction}

Snow or rock avalanches in nature are generally regarded as a class of massive slide phenomena involving gravity and density currents. These flows slide down a slope as a mixture of solid and fluid, and exhibit various patterns and complicated inner structures. For instance, powder snow avalanches are roughly divided into two structural regions according to field observations [1,2]: dense and dilute regions. The former is formed in the vicinity of a slope, whereas the latter develops above the dense region. In general, the dense region consists of coarse or high-density particles that are packed densely in the flow. The dilute region (the powder cloud) comprises fine particles or low-density particles that are suspended in the air. The driving force of both flows is gravity. However, the resisting force is different: inner and basal frictions govern the dense flow, whereas drag force governs the powder cloud. Therefore, these resisting forces lead to a major difference in the structure and dynamics.

A number of laboratory experiments on dense flow have been performed [3-9]. Dense granular flows consisting of monodisperse glass beads form the fingering pattern at the front and the streaky 
structure at the surface $[4,5]$, thus leading to flow instability. In these flow patterns, the recirculation of particles at the front is caused by the velocity profile with flow height, and granular convection is confirmed inside the streaky structure. Moreover, polydisperse particles result in segregation depending on the particle size $[7,8]$. Because basal friction is a key factor to understand the mechanism of dense flow, it is measured in snow particle experiments [6]. Numerical models on dense flow are categorized into two primary types: the discrete element method (DEM) $[10,11]$ and the continuum model $[7,8,12-16]$. Recently, the granular continuum model was combined with the governing equation for the fluid, and a two-phase flow model was developed for wet granular flows [16].

Experiments on powder clouds have been conducted with regard to various materials, situations, and scales to adjust several dimensionless numbers [17-25]. The typical value of the particle Reynolds number $R e_{\mathrm{p}}$ in natural powder cloud in snow avalanches is $\sim 3000$. This value is higher than the experimental values (see [22]). However, polystyrene-air flows with an $R e_{\mathrm{p}}$ value of $\sim 150$ can reproduce the cloud pattern except in cases of particles in turbulent eddies [22-25]. In these experiments, the head-tail structure and wavy pattern are formed due to flow instability. Here, the head refers to a large dense particle cluster at the moving front of the granular flow, whereas the tail denotes the thin layer behind the head. The recirculation of particles occurs at the head $[21,23,25]$, and the direction is opposite to the recirculation of dense flow [4]. These dynamics are observed at actual-scale granular flows consisting of low-density ping pong balls $\left(\rho_{\mathrm{p}} \approx 11 \mathrm{~kg} \mathrm{~m}^{-3}\right)[19,20]$. Furthermore, a similar cloud is confirmed for falling particles in a viscous fluid $\left(R e_{\mathrm{p}} \ll 1\right)$, although the particles do not move on the slope [26].

Many studies depict powder clouds as solid-gas two-phase flow models [18,27-34] as the particle is significantly affected by the fluid. This type of model is suitable for the large-scale dynamics of actual mountain topographies. However, experiments on powder clouds use low-density particles such as polystyrene particles and ping pong balls. Therefore, a different approach, such as a particle-based model, is required to understand the mechanism of the powder cloud. The authors of [19] developed a numerical model that coupled the DEM for the particles and the Reynolds-averaged Navier-Stokes equations for the fluid. However, this model could not reproduce the flow pattern of the experiments. Thus, they suggested considering the strong interaction between each particle trajectory and the fluid.

In this study, we attempt to fill in the above-mentioned gaps by proposing a simple particle model for granular flow consisting of low-density particles to qualitatively understand the time evolutions of frontal speed and head shape. In Section 2, we describe the model and subsequently explain the set-up of the numerical simulations with the model. Section 3 presents the simulation results regarding flow pattern and speed. Section 4 discusses the shapes of the flow patterns.

\section{Methods}

\subsection{Concept of the Proposed Model}

We propose a simple particle model for low-density granular flow based on the equation of motion for particles. Realistically, the granular flows of natural phenomena are roughly affected by physical factors, namely, gravity and basal and inner frictions. The motion of each particle in the flow is also affected by the particle size and turbulence intensity. Thus, the dynamics of granular flow are complex, and understanding the mechanism becomes difficult. Our model aims to significantly simplify the complicated behavior of low-density granular flows interacting with the ambient fluid. Although our model is not realistic at this time, we aim to reproduce the flow patterns found in previous experiments with ping pong balls and polystyrene particles [20,23].

As a simple case, we consider that a low-density granular flow slides on a slope at an angle steeper than the angle of repose (Figure 1). This model is based on three basic assumptions for the simplification of the modeling: (i) The granular flow consists of hard spherical particles composed of a material of low specific density. (ii) Only the translational motion of the particles is considered (i.e., the rotational motion is ignored). (iii) Three types of forces act on the particles: (a) gravity as the driving 
force for granular flow, (b) the repulsive force between the particles due to particle collision, and (c) the drag force due to the ambient fluid. Figure 1 shows the schematic of this model, where $\theta$ is the incline angle, the $x$-axis is the inclination direction, the $y$-axis is the lateral direction, and the $z$-axis is perpendicular to the slope.

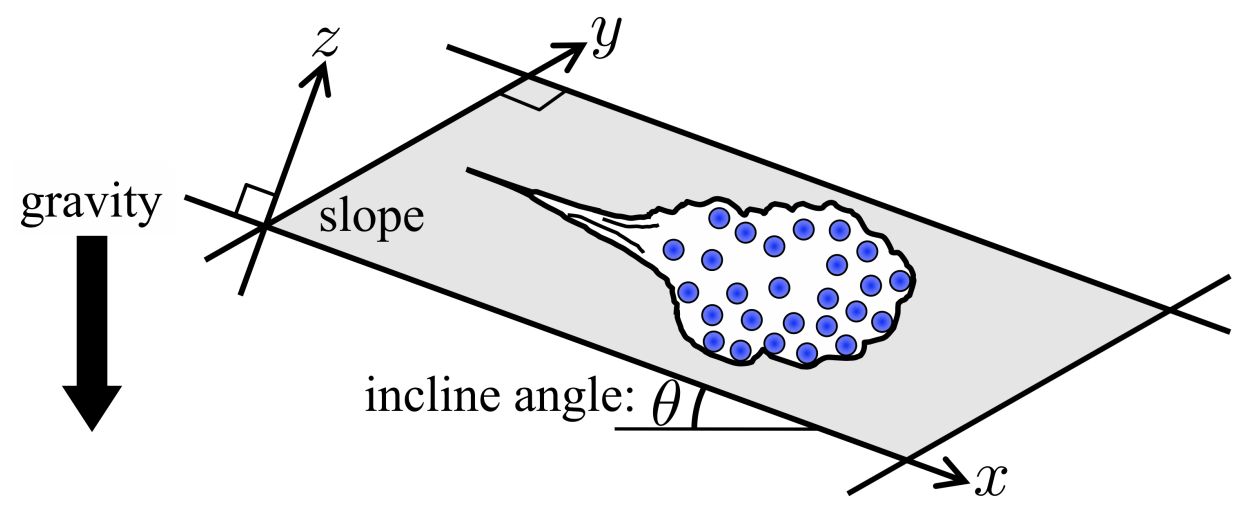

Figure 1. Schematic of a simple particle model. The $x-y$ plane is parallel to the slope with the incline angle of $\theta$ ( $x$ : inclination direction; $y$ : lateral direction). The $z$-axis is perpendicular to the slope.

\subsection{Particle Model}

To derive the governing equation for the particles, we formulate the force acting on the $i$ th particle $\boldsymbol{F}_{i}=\boldsymbol{F}_{i}^{g}+\boldsymbol{F}_{i}^{r}+\boldsymbol{F}_{i}^{d}$, where $\boldsymbol{F}_{i}^{g}$ is gravity, $\boldsymbol{F}_{i}^{r}$ is the repulsive force between the particles, and $\boldsymbol{F}_{i}^{d}$ is the drag force due to the ambient fluid. Hereinafter, the radius, coordinate, and velocity of the $i$ th particle are denoted by $a_{i}, \boldsymbol{r}_{i}$, and $\boldsymbol{v}_{i}$, respectively, and the true density of the particles is denoted by $\rho_{\mathrm{p}}$.

Gravity $\boldsymbol{F}^{g}$ : The gravity in the vertical direction of the $i$ th particle $F_{i}^{g}$ is calculated by considering the density difference between the particle and fluid. Subsequently, it is divided into the $x$ and $z$ components depending on the incline angle $\theta$, as follows:

$$
\begin{aligned}
\boldsymbol{F}_{i}^{g} & =F_{i}^{g}\left(\begin{array}{c}
-\sin \theta \\
0 \\
\cos \theta
\end{array}\right)=F_{i}^{g} e_{\theta}, \\
F_{i}^{g} & =-V_{i} g\left(\rho_{\mathrm{p}}-\rho_{\mathrm{f}}\right), \\
V_{i} & =\frac{4}{3} \pi a_{i}^{3},
\end{aligned}
$$

where $e_{\theta}$ is the transformation vector from the standard coordinate system into the incline coordinate system, as shown in Figure $1, V_{i}$ is the volume of the $i$ th particle, $g$ is the gravitational acceleration, and $\rho_{\mathrm{f}}$ is the density of the fluid.

Repulsive force $\boldsymbol{F}^{r}$ : Because the rotational motion of the particles is ignored, only the normal force of collision between the $i$ th and $j$ th particles is calculated as the repulsive force of the $i$ th particle $\boldsymbol{F}_{i}^{r}$ (and $\boldsymbol{F}_{j}^{r}$ ), which is represented by the elastic spring force $F_{i}^{r}$ as follows,

$$
\begin{aligned}
\boldsymbol{F}_{i}^{r} & =F_{i}^{r} \frac{\boldsymbol{r}_{j}-\boldsymbol{r}_{i}}{r_{i j}}=F_{i}^{r} \boldsymbol{n}_{i j}, \\
F_{i}^{r} & =-k_{n} \delta_{i j}, \\
\delta_{i j} & =\left\{\begin{array}{cl}
a_{i}+a_{j}-r_{i j} & \left(r_{i j} \leq a_{i}+a_{j}: \text { contact }\right) \\
0 & \left(r_{i j}>a_{i}+a_{j}: \text { non-contact }\right) .
\end{array}\right.
\end{aligned}
$$

Here, $r_{i j}=\left|\boldsymbol{r}_{j}-\boldsymbol{r}_{i}\right|$ is the interparticle distance and $\boldsymbol{n}_{i j}$ is the unit vector joining the centers of two particles. Further, $k_{n}$ is the linear spring constant and $\delta_{i j}$ is the overlap between two particles. For 
the collision between a particle and the slope, we calculate the overlap distance between them. Then, the normal repulsive force acting on the particle is determined using Equation (2b).

Drag force $\boldsymbol{F}^{d}$ : In this model, we estimate the drag force from the fluid velocity field generated by the particle motion. Concretely, the ambient fluid is assumed to be Stokes flow (i.e., we assume a low Reynolds number; $R e \ll 1$ ), although the particle inertia is not negligible and the turbulent eddy occurs in the actual granular flow. The Stokes flow containing two spherical particles can be solved theoretically when a sufficient distance exists between two particles. The exact solution is known as the Rotne-Prager tensor $\mathbf{J}(\boldsymbol{r})$ [35]. This tensor can solve the fluid velocity field based on the velocity of the $j$ th particle. The fluid velocity at the coordinate of the $i$ th particle is added to the velocity of the $i$ th particle as the induced velocity, because the particle completely follows the fluid flow in Stokes flow. Using Stokes' drag (Equation (3a)) and the Rotne-Prager tensor (Equation (3c)), we express the interparticle interaction through the ambient fluid, that is, the drag force of the $i$ th particle $F_{i j}^{d}$ is generated by the force acting on the $j$ th particle $\boldsymbol{F}_{j}^{g+r}=\boldsymbol{F}_{j}^{g}+\boldsymbol{F}_{j}^{r}$, as follows:

$$
\begin{aligned}
\boldsymbol{F}_{i j}^{d} & =6 \pi \mu a_{i} \boldsymbol{u}_{i}(j), \\
\boldsymbol{u}_{i}(j) & =\frac{1}{8 \pi \mu} \mathbf{J}\left(\boldsymbol{r}=\boldsymbol{r}_{i}-\boldsymbol{r}_{j}\right) \cdot \boldsymbol{F}_{j}^{g+r}, \\
\mathbf{J}(\boldsymbol{r}) & =\frac{1}{r_{j i}}\left[\boldsymbol{I}+\frac{\boldsymbol{r} \boldsymbol{r}}{r_{j i}^{2}}+\frac{2}{3}\left(\frac{a_{j}}{r_{j i}}\right)^{2}\left(\boldsymbol{I}-3 \frac{\boldsymbol{r} \boldsymbol{r}}{r_{j i}^{2}}\right)\right] .
\end{aligned}
$$

In these equations, $\mu$ is the viscosity coefficient of the fluid, $u_{i}(j)$ is the velocity induced by the $j$ th particle at the coordinate of the $i$ th particle (Figure 2), and $I$ is the unit tensor. Particularly, $r r$ in Equation (3c) denotes the tensor corresponding to $\boldsymbol{I}$. For instance, $r r_{x y}=\left(x_{i}-x_{j}\right)\left(y_{i}-y_{j}\right)$.

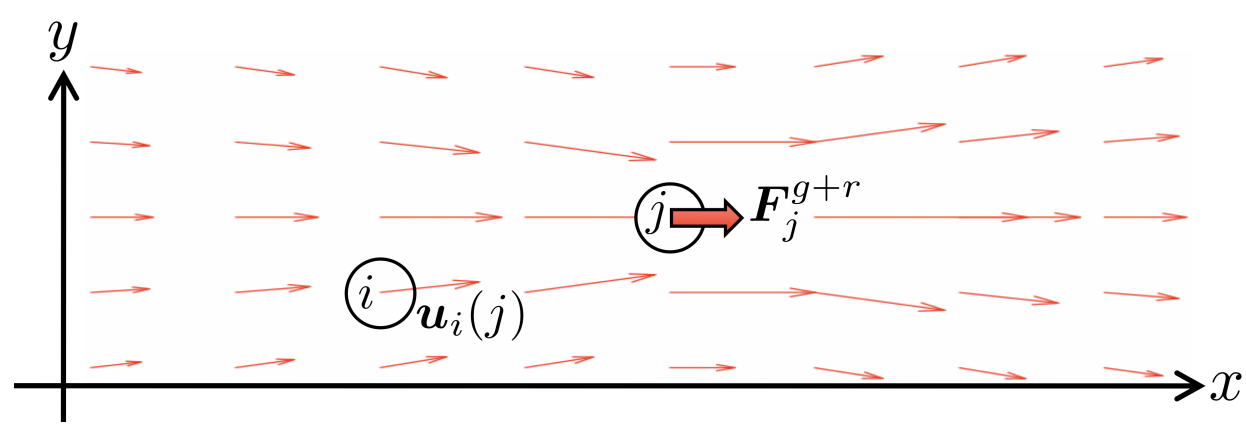

Figure 2. Induced velocity field $u(j)$ generated by gravity and the repulsive force of the $j$ th particle, $\boldsymbol{F}_{j}^{g+r}$, using Equation (3b). According to the force direction, the ambient fluid is pushed and pulled as shown by the arrows. The induced velocity at the $i$ th particle is expressed as $\boldsymbol{u}_{i}(j)$.

According to the assumption for the drag force (i.e., Stokes flow), we derive the governing equation for the $i$ th particle from Stokes' drag as follows,

$$
\begin{aligned}
\boldsymbol{v}_{i} & =\frac{\boldsymbol{F}_{i}}{6 \pi \mu a_{i}}=\frac{\boldsymbol{F}_{i}^{g+r}}{6 \pi \mu a_{i}}+\sum_{j \neq i}^{N} \boldsymbol{u}_{i}(j), \\
\frac{d \boldsymbol{r}_{i}}{d t} & =\boldsymbol{v}_{i},
\end{aligned}
$$

where $N$ is the number of particles. In Equation (4a), the second term indicates the long-range interaction between particles through the ambient fluid, which resembles the asymmetric potential (Figure 2). A limitation of this model is that the Rotne-Prager tensor overestimates the interaction between particles when they are close to each other (its condition is out of the theory [35]). The 
simulation of granular flow with this tensor is not realistic, but it could be helpful to understand the time evolution of granular flow strongly affected by the drag force.

\subsection{Simulation Setup}

The numerical modeling for the low-density granular flow is performed in a three-dimensional space, as shown in Figure 1. However, we use the time complexity of $O\left(\mathrm{~N}^{2}\right)$ in our model to calculate the drag force $\boldsymbol{F}_{i j}^{d}$ in Equation (3). The repulsive force $\boldsymbol{F}_{i}^{r}$ in Equation (2) additionally requires the small timestep $d t$ used in the numerical simulation. Therefore, this model is unsuitable for calculations involving a large number of particles. In this study, the number of particles is set as $N=2000$. Subsequently, numerical simulations are conducted in two-dimensional planes: the $x-y$ plane parallel to the slope and the $x-z$ plane perpendicular to the slope.

Figure 3 shows three different types of initial conditions for the particles in the $x-y$ and $x-z$ planes: (a) circular shape, (b) rectangular shape, and (c) triangular shape. Here, the incline angle is fixed as $\theta=45^{\circ}$. These shapes are determined by reference to previous experiments with polystyrene particles [23] although the size is small in comparison to the work in [23] because of the small $N$. The circle radius $R$, rectangle width $W$, and triangle depth $D$ are set as constant values depending on the particle size used in the simulations, as shown later. Particularly, in the case of Figure $3 b$, the length of the rectangle in the $x$ direction is fixed as 10 particles. In each shape, 2000 particles are packed randomly without overlap.

(a)

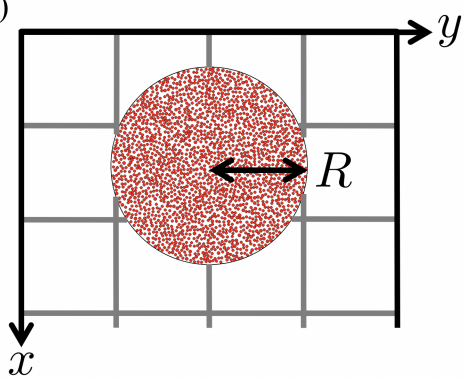

(b)

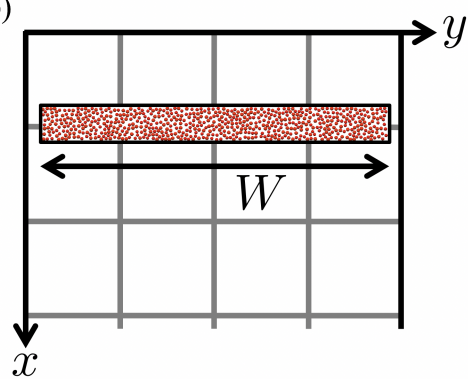

(c)

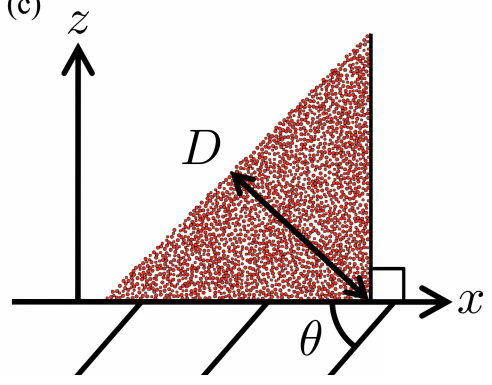

Figure 3. The three different types of initial conditions for particles in two-dimensional planes are as follows, (a) circle in the $x-y$ plane, (b) rectangle in the $x-y$ plane, and (c) triangle in the $x-z$ plane. The $x-y$ plane is parallel to the slope, whereas the $x-z$ plane is the vertical cross section on the slope, where $\theta=45^{\circ}$ is the incline angle. In each initial condition, the circle radius $R$, rectangle width $W$, and triangle depth $D$ are constant depending on the particle size, such that the particle volume fraction $\Phi_{\mathrm{p}}$ of 2000 particles is approximately 0.5 .

Regarding the specific materials of the particle and fluid, we assume that the granular flow consists of low-density particles, such as polystyrene in air, to allow a qualitative comparison with the previous experiments [23]. The particle density and linear spring constant for the repulsive force are given as $\rho_{\mathrm{p}}=20 \mathrm{~kg} \mathrm{~m}^{-3}$ and $k_{n}=10 \mathrm{~N} \mathrm{~m}^{-1}$, respectively, whereas the density and viscosity coefficient of the fluid are $\rho_{\mathrm{f}}=1.2 \mathrm{~kg} \mathrm{~m}^{-3}$ and $\mu=1.82 \times 10^{-5} \mathrm{~Pa} \mathrm{~s}$, respectively. We determine the value of $k_{n}$ so that the overlap distance between particles can be small. As the control parameter, we vary only the particle radius, such that $a_{\mathrm{p}}=1,2.5$, and $5 \mathrm{~mm}$ (we consider three values). To avoid the crystallization of particles, particles with a polydispersity of $\pm 5 \%$ are used in simulations.

The sizes of the initial shapes $R, W$, and $D$, as shown in Figure 3, were determined depending on $a_{\mathrm{p}}=1,2.5$, and $5 \mathrm{~mm}: R=0.065,0.17$, and $0.34 \mathrm{~m} ; W=0.35,0.85$, and $1.7 \mathrm{~m}$; and $D=$ $0.115,0.3$, and $0.6 \mathrm{~m}$. These sizes normalized by $a_{\mathrm{p}}$ are $\tilde{R}=65-68, \tilde{W}=340-350$, and $\tilde{D}=115-120$, respectively. Further, the particle volume fraction is approximately 0.5 . We perform 20 simulations by changing the initial position of the particles foreach initial shape and each particle radius. 


\subsection{Nondimensionalization of the Proposed Model}

To provide consistent interpretation of numerical simulations, we conduct the nondimensionalization of our model before the results. First, we derived the speed of the single particle $v_{\mathrm{s}}$ in the case of $N=1$; that is, this is obtained by referring to gravity alone. The particle coordinates $r$, particle velocity $v$, and time $t$ are normalized using the particle radius $a_{\mathrm{p}}$ and $v_{\mathrm{S}}$ as follows,

$$
\begin{gathered}
\tilde{\boldsymbol{r}}=\frac{\boldsymbol{r}}{a_{\mathrm{p}}}, \tilde{\boldsymbol{v}}=\frac{\boldsymbol{v}}{v_{\mathrm{s}}}, \tilde{t}=\frac{t}{a_{\mathrm{p}} / v_{\mathrm{s}}}, \\
v_{\mathrm{s}}=\frac{2}{9} \frac{g\left(\rho_{\mathrm{p}}-\rho_{\mathrm{f}}\right) \sin \theta}{\mu} a_{\mathrm{p}}^{2} .
\end{gathered}
$$

Next, the governing equations for the ith particle (Equation (4)) are rewritten as follows,

$$
\begin{aligned}
\tilde{\boldsymbol{v}}_{i} & =\frac{1}{6 \pi} \frac{\boldsymbol{F}_{i}^{g+r}}{\mu a_{\mathrm{p}} v_{\mathrm{s}}}+\sum_{j \neq i}^{N} \frac{\boldsymbol{u}_{i}(j)}{v_{\mathrm{s}}}=\frac{1}{6 \pi} \tilde{\boldsymbol{F}}_{i}^{g+r}+\sum_{j \neq i}^{N} \tilde{\boldsymbol{u}}_{i}(j), \\
\frac{d \tilde{\boldsymbol{r}}_{i}}{d \tilde{t}} & =\tilde{\boldsymbol{v}}_{i}
\end{aligned}
$$

where $\tilde{\boldsymbol{F}}_{i}^{g+r}=\tilde{\boldsymbol{F}}_{i}^{g}+\tilde{\boldsymbol{F}}_{i}^{r}$ is the sum of the dimensionless gravity and dimensionless repulsive force of the $i$ th particle, and $\tilde{\boldsymbol{u}}_{i}(j)$ is the dimensionless induced velocity generated by the $j$ th particle at the coordinate of the $i$ th particle. Furthermore, the dimensionless drag force $\tilde{\boldsymbol{F}}_{i j}^{d} \equiv \boldsymbol{F}_{i j}^{d} /\left(\mu a_{\mathrm{p}} v_{\mathrm{s}}\right)$ and $\tilde{\boldsymbol{u}}_{i}(j)$ are expressed using Equation (3) as follows,

$$
\begin{aligned}
\tilde{\boldsymbol{F}}_{i j}^{d} & =6 \pi \tilde{\boldsymbol{u}}_{i}(j), \\
\tilde{\boldsymbol{u}}_{i}(j) & =\frac{1}{v_{\mathrm{s}}} \frac{1}{8 \pi \mu} \mathbf{J}(\boldsymbol{r}) \cdot \boldsymbol{F}_{j}^{g+r} \\
& =\frac{1}{8 \pi} \mathbf{J}(\tilde{\boldsymbol{r}}) \cdot \tilde{\boldsymbol{F}}_{j}^{g+r} .
\end{aligned}
$$

In this equation, because the dimension of the Rotne-Prager tensor $\mathbf{J}(\boldsymbol{r})$ is the inverse of $\boldsymbol{r}$, we obtain the equality $\mathbf{J}(\boldsymbol{r})=\mathbf{J}(\tilde{\boldsymbol{r}}) / a_{\mathrm{p}}$. The dimensionless parameter is include in $\tilde{\boldsymbol{F}}_{i}^{g+r}$ in Equations (6a) and (7b).

Finally, the dimensionless gravity $\tilde{\boldsymbol{F}}_{i}^{g}$ is expressed using Equation (1) as follows,

$$
\begin{aligned}
\tilde{\boldsymbol{F}}_{i}^{g} & =-\frac{1}{\mu a_{\mathrm{p}} v_{\mathrm{s}}} V_{i} g\left(\rho_{\mathrm{p}}-\rho_{\mathrm{f}}\right) e_{\theta} \\
& =-\frac{6 \pi}{\sin \theta} e_{\theta}
\end{aligned}
$$

where $\theta$ is the incline angle of the slope, and $e_{\theta}$ is the vector defined in Equation (1a). The dimensionless repulsive force $\tilde{\boldsymbol{F}}_{i}^{r}$ is also expressed using Equation (2) as follows:

$$
\begin{aligned}
\tilde{\boldsymbol{F}}_{i}^{r} & =-\frac{1}{\mu a_{\mathrm{p}} v_{\mathrm{s}}} k_{n} \delta_{i j} \boldsymbol{n}_{i j} \\
& =-\frac{k_{n} \delta_{i j}}{V_{i g}\left(\rho_{\mathrm{p}}-\rho_{\mathrm{f}}\right)} \frac{6 \pi}{\sin \theta} \boldsymbol{n}_{i j} \\
& =-\frac{F_{i}^{r}}{F_{i}^{g}} \frac{6 \pi}{\sin \theta} \boldsymbol{n}_{i j} \\
& =-\gamma \frac{6 \pi}{\sin \theta} \boldsymbol{n}_{i j}
\end{aligned}
$$


where $\gamma$ is the dimensionless parameter in our model. The physical meaning of $\gamma$ is the ratio of the excluded volume effect between particles divided by gravity. We substitute the values used for the simulations in $\gamma$ and assume $\delta_{i j}=0.01 a_{\mathrm{p}}$. Then, $\gamma$ is calculated as 130,20 , and $5\left(a_{\mathrm{p}}=1,2.5\right.$, and $5 \mathrm{~mm}$ ). It means that the repulsive force is larger than gravity in our simulation.

\section{Results}

\subsection{Circular Shape in the $x-y$ Plane}

The typical time evolutions of the granular flow in the circular shape are shown in Figure 4. Here, the particle radius is $a_{\mathrm{p}}=5 \mathrm{~mm}$. In the early stage of the simulations, the particle located at the central part of the circle migrates rapidly to the moving front of the granular flow as time progresses. Consequently, the initial uniform distribution of particles shifts to the local distribution at the moving front of the granular flow (Figure $4 a, b)$. This pattern of particle aggregation appears to be a single head resembling a crescentic form, similar to the pattern of a low-density granular flow [20,23]. After the formation of the head, the particle moves away gradually from the aggregated part. Subsequently, the head shrinks with time (Figure $4 \mathrm{c}, \mathrm{d}$ ). This head formation process is also confirmed for the other particle radiuses although the time required for the head formation is different.
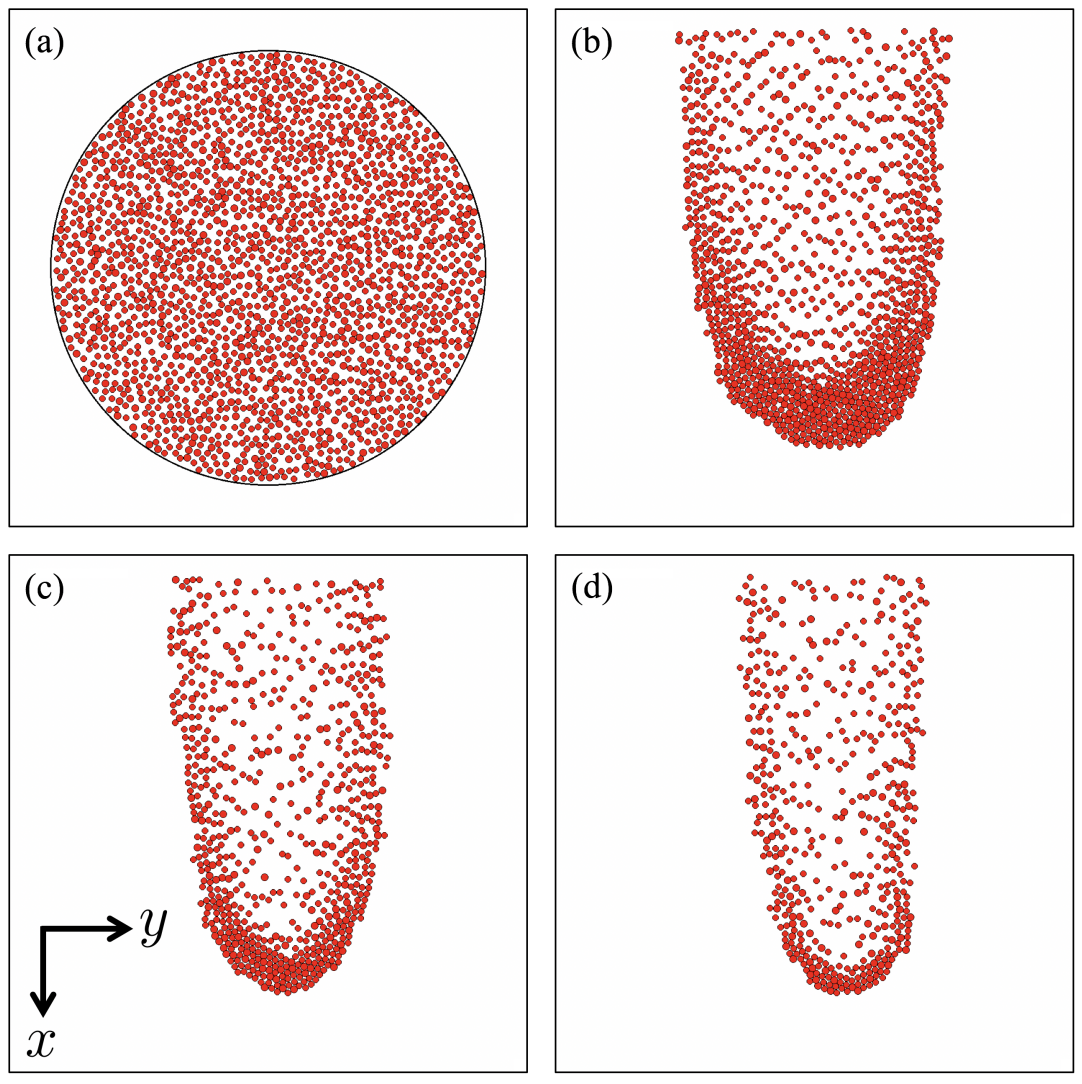

Figure 4. Time evolution of single head formation with $a_{\mathrm{p}}=5 \mathrm{~mm}$ in the circular setup: (a) $\tilde{t}=0$, (b) $\tilde{t}=7.96,(\mathbf{c}) \tilde{t}=19.5$, and $(\mathbf{d}) \tilde{t}=39.8$.

To verify the key factor for the head formation, we perform the simulation without the drag force. In this case, the initial pattern is maintained because the basal friction and particle inertia are ignored in our model. Therefore, the drag force generates a long-range interaction between particles through the velocity induced by the ambient fluid $\boldsymbol{u}_{i}(j)$ in Equation (3), which is essential to form the head. The reason for the head formation, namely owing to the induced velocity, is clear. In the initial stage of the simulations (Figure 4a), the induced velocity is high at the central part because it is inversely 
proportional to the interparticle distance. Therefore, particle aggregation occurs in the moving front of the granular flow, and the aggregation migrates at a faster speed.

For the migration speed of the granular flow, we measure the frontal speed $v_{\mathrm{f}}$ from the displacement in the $x$ direction of the forefront particle position. In our model, $v_{\mathrm{f}}$ is defined as follows,

$$
v_{\mathrm{f}}=\frac{\left[\max x_{i}(t)-\max x_{i}(t-d t)\right]}{d t},
$$

where $d t$ is the time step. Consequently, the frontal speed is normalized by the speed of the single particle (Equation (5b)) as $\tilde{v}_{\mathrm{f}}=v_{\mathrm{f}} / v_{\mathrm{s}}$. Because $v_{\mathrm{s}}$ increases with $a_{\mathrm{p}}$, the smaller time step $d t$ is required for the calculation of the repulsive force in the larger $a_{\mathrm{p}}$. The numerical simulations continue until the granular flow reaches the state shown in Figure $4 \mathrm{~d}: t_{\mathrm{end}}=20,10$, and $5 \mathrm{~ms}\left(a_{\mathrm{p}}=1,2.5\right.$, and $\left.5 \mathrm{~mm}\right)$.

Figure 5 a shows the temporal variations in the normalized frontal speeds $\tilde{v}_{\mathrm{f}}(\tilde{t})$ measured at different particle radii $a_{\mathrm{p}}$. The solid curve and thin band denote the mean and standard deviation of 20 simulations, respectively. The standard deviation is small compared to the mean even though the size distribution and initial position of the particles are changed. In all cases, $\tilde{v}_{\mathrm{f}}$ increases significantly in the early stage and subsequently decreases gradually with time because of the head shrinkage. Additionally, $\tilde{v}_{\mathrm{f}}$ ranges from 30 to 70, independent of $a_{\mathrm{p}}$. Therefore, the head size (i.e., number of particles) causes the change in $\tilde{v}_{\mathrm{f}}$ (Figures 4 and $5 \mathrm{a}$ ). The head size is discussed in detail in Section 4.2.
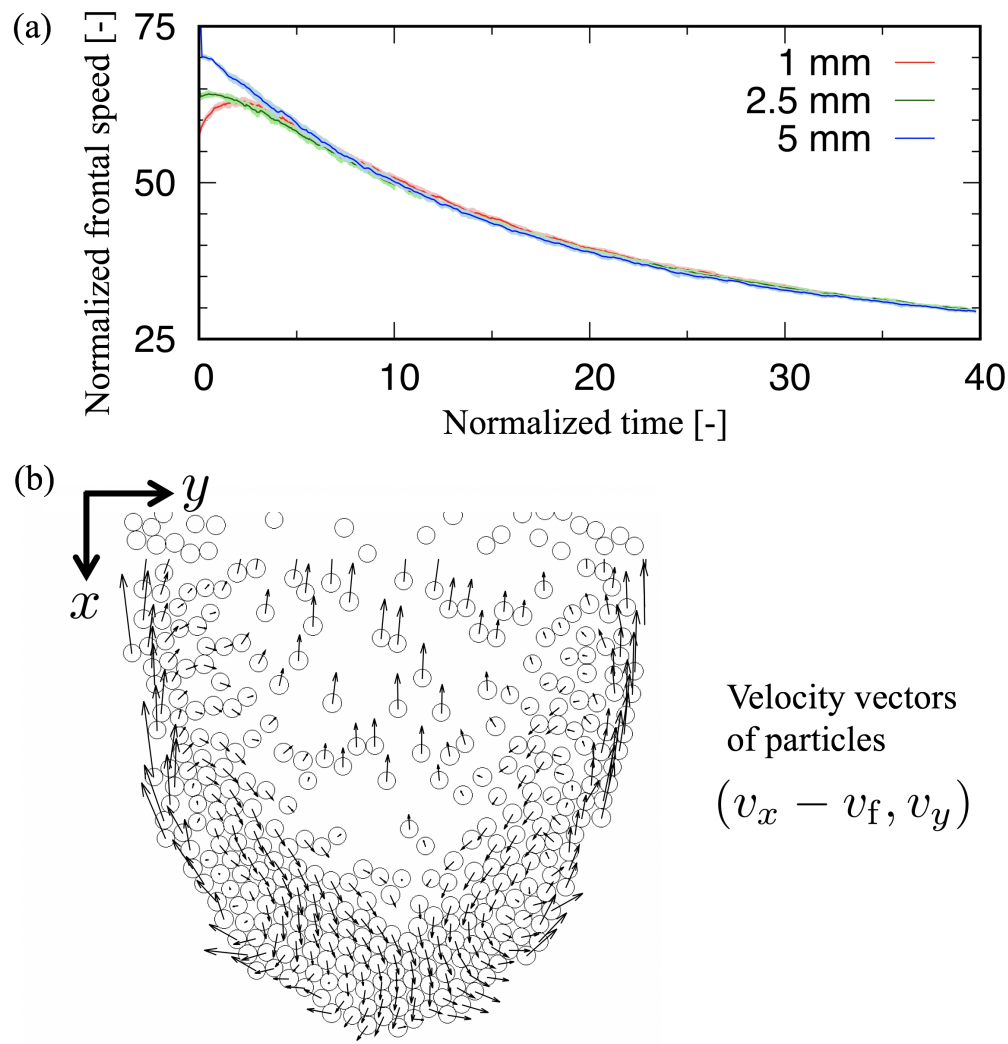

Figure 5. Temporal variations in particle velocity in the circular set-up: (a) normalized frontal speed of the single head $\tilde{v}_{\mathrm{f}}(\tilde{t})$ at different particle radiuses $a_{\mathrm{p}}$ and $(\mathbf{b})$ velocity vector of particles in the head, as shown in Figure 4c. (a) The mean and standard deviation of 20 simulations are denoted by a solid curve and thin band, respectively.

The movements of the particles in the head can be visualized by their respective velocity vectors (Figure 5b). This particle location corresponds to that in Figure 4c; that is, $\tilde{t}=19.5$ and $a_{\mathrm{p}}=5 \mathrm{~mm}$. To study the particle velocity relative to the frontal speed of the granular flow $v_{\mathrm{f}}$, the $x$ component of 
the vectors is denoted by $v_{x}-v_{\mathrm{f}}$, where $v_{x}$ is the $x$ component of the particle velocity. Consequently, a granular vortex convection is observed in the head as well as the particles away it. In particular, the particle at the rear of the head moves inward to the forefront of the head, whereas the particle at the front of the head is pushed outward. Some particles return to the head again. However, most of the particles are left behind. Thus, the head shrinks with time.

\subsection{Rectangular Shape in the $x-y$ Plane}

The typical time evolutions of granular flow in the rectangular shape are shown in Figure 6a-c. Here, the particle radius is $a_{\mathrm{p}}=5 \mathrm{~mm}$. The simulation time at each $a_{\mathrm{p}}$ is the same as that for the circular shape: $t_{\mathrm{end}}=20,10$, and $5 \mathrm{~ms}\left(a_{\mathrm{p}}=1,2.5\right.$, and $\left.5 \mathrm{~mm}\right)$. In the early stage of the simulations, the initial straight shape at the moving front of the granular flow is maintained although the particle moves forward slowly to the front due to the long-range interaction between the particles via the drag force. Subsequently, the straight shape with particle aggregation deforms into a wavy pattern (Figure 6b); that is, flow instability occurs. This destabilization of the moving front is caused by the inhomogeneous distribution of the size and coordinates in the initial condition of the particles (Figure 6a). Because the aggregation is faster than the other parts, the wavy pattern elongates in a downward direction. Eventually, the multihead structure is formed from the straight shape (Figure 6c).
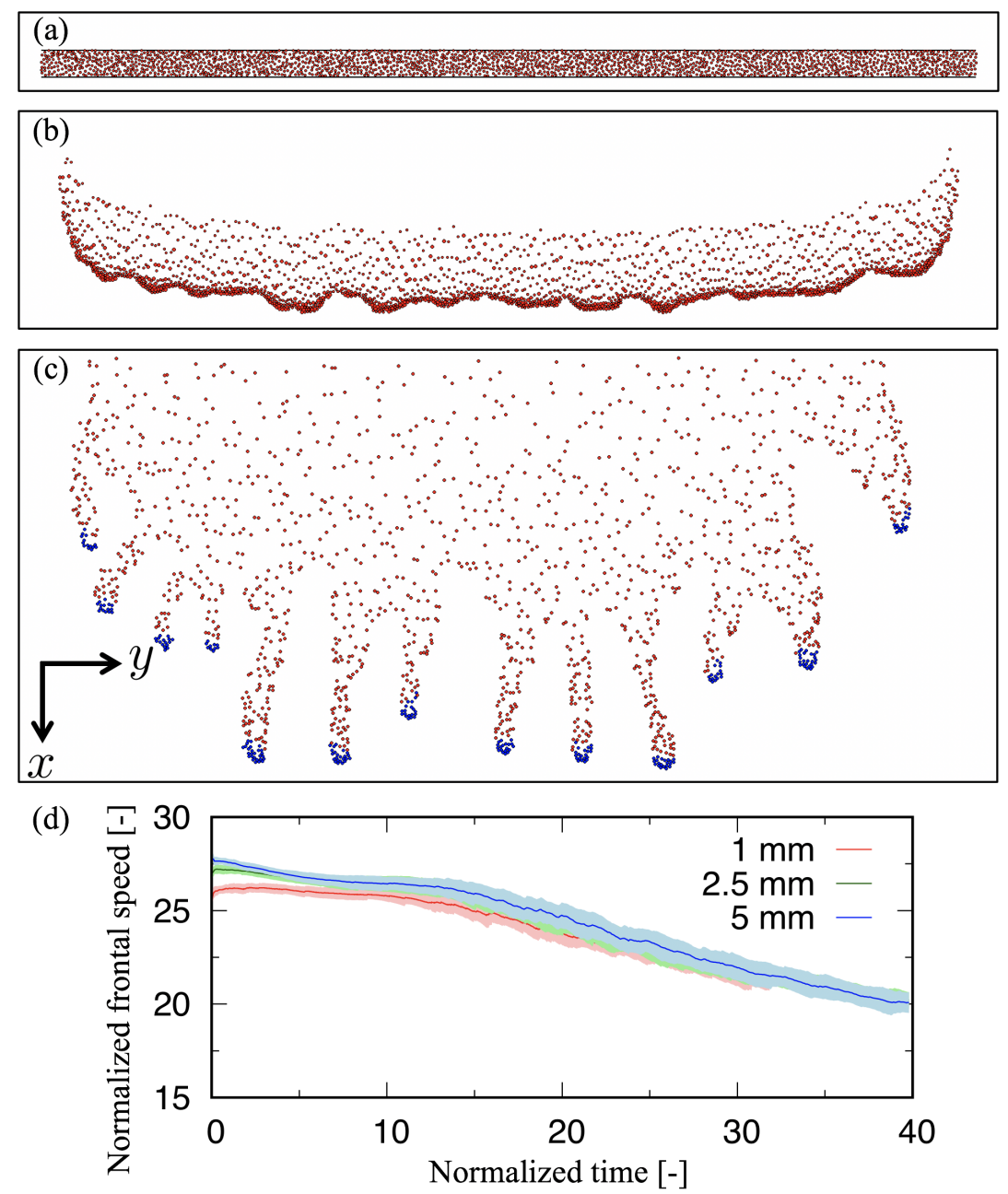

Figure 6. Flow instability from the straight shape to the wavy pattern with $a_{\mathrm{p}}=5 \mathrm{~mm}$ in the rectangular set-up: (a) $\tilde{t}=0$, (b) $\tilde{t}=7.96$, and (c) $\tilde{t}=39.8$. (c) The blue particle belongs to the aggregated part detected as the head, according to Equation (11b). (d) Three normalized frontal speeds $\tilde{v}_{\mathrm{f}}(\tilde{t})$ are shown at $a_{\mathrm{p}}=1,2.5$, and $5 \mathrm{~mm}$. The mean and standard deviation of 20 simulations are denoted by the solid curve and thin band, respectively. 
Figure $6 \mathrm{~d}$ shows the front speed in the $x$ direction normalized by the speed of the single particle, $\tilde{v}_{\mathrm{f}}(\tilde{t})$, at each particle radius $a_{\mathrm{p}}$. The mean and standard deviation of 20 simulations are denoted by the solid curve and thin band, respectively. In all cases, $\tilde{v}_{\mathrm{f}}$ decreases gradually in the early stage. Subsequently, the decreasing rate of $\tilde{v}_{\mathrm{f}}$ tends to be relatively high. Additionally, $\tilde{v}_{\mathrm{f}}$ ranges from 20 to 28 independent of $a_{\mathrm{p}}$, and the formation of the multihead structure is confirmed at the end of each simulation (Figure 6c). The formed pattern (number of heads and head size) differs depending on the initial condition. However, the wavelength reproducibility is good. This point is discussed in detail in Section 4.2.

In Figure $6 \mathrm{~d}$, the rate of decrease in $\tilde{v}_{\mathrm{f}}$ is linked with the flow pattern, as shown in Figure 6a-c. For example, during the deformation from the straight shape (Figure 6a) to the wavy pattern (Figure 6b), the number of particles constituting the aggregation remains nearly unchanged. Thus, $\tilde{v}_{\mathrm{f}}$ is maintained in the early stage of the simulations. Simultaneously, the number of particles decreases locally with the elongation of the heads from the wavy pattern. Consequently, $\tilde{v}_{\mathrm{f}}$ decreases at a relatively high rate after the middle stage of the simulations.

\subsection{Triangular Shape in the $x-z$ Plane}

The typical time evolutions of the granular flow in the triangular shape are shown in Figure 7a,b. Here, the incline angle and particle radius are $\theta=45^{\circ}$ and $a_{\mathrm{p}}=5 \mathrm{~mm}$, respectively. The simulation time at each $a_{\mathrm{p}}$ is set as $t_{\text {end }}=20,10$, and $5 \mathrm{~ms}\left(a_{\mathrm{p}}=1,2.5\right.$, and $\left.5 \mathrm{~mm}\right)$. Because the particles are up in the air initially (Figure 3c), they immediately accumulate near the slope according to the gravity. Subsequently, a large-scale cluster consisting of particles emerges from the accumulation, and moves down a slope at a high speed (Figure 7a). This pattern appears to be the head-tail structure, where the thickness in the $z$ direction of the front part is more than twice that of the rear part. This profile is similar to the flow height of the experiments with ping pong balls [19]. After the head-tail structure is formed, the head shrinks with time (Figure 7b).
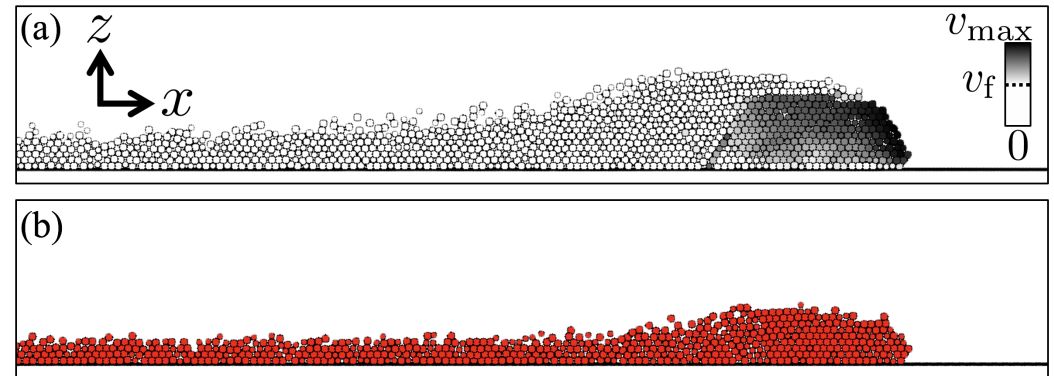

(c)

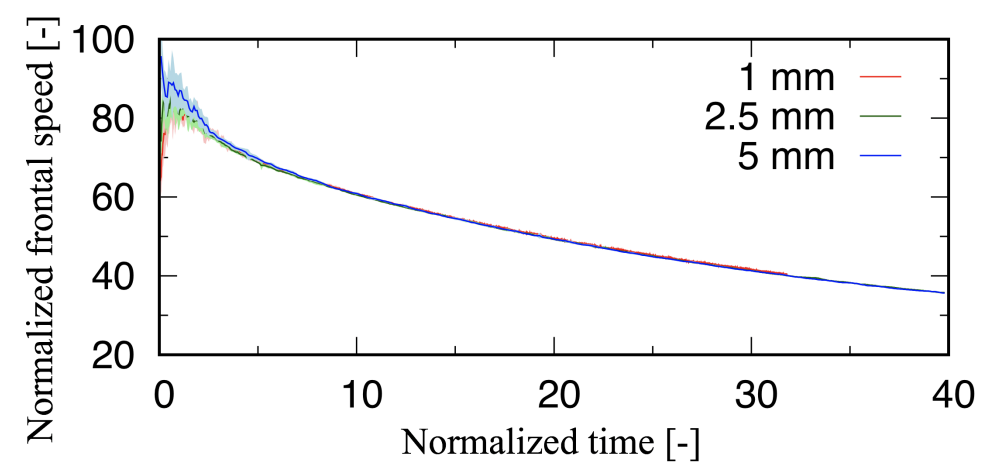

Figure 7. Formation of head-tail structure with $a_{\mathrm{p}}=5 \mathrm{~mm}$ in the triangular set-up: (a) $\tilde{t}=15.9$, and (b) $\tilde{t}=39.8$. (a) The gray scale denotes the $x$ component of the particle velocity. The white particles are slower compared to the frontal speed $v_{\mathrm{f}}$, whereas the gray particles are faster compared to $v_{\mathrm{f}}$. Here, $v_{\mathrm{f}}$ is calculated from the particle on the slope (i.e., the first layer). (c) Three normalized frontal speeds $\tilde{v}_{\mathrm{f}}(\tilde{t})$ are shown at $a_{\mathrm{p}}=1,2.5$, and $5 \mathrm{~mm}$. The mean and standard deviation of 20 simulations are denoted by a solid curve and thin band, respectively. 
In the $x-z$ plane, the front speed in the $x$ direction $v_{\mathrm{f}}$ is measured according to the displacement of the forefront particle on the slope (i.e., the first layer). Figure 7c shows the normalized front speed $\tilde{v}_{\mathrm{f}}(\tilde{t})$ at different particle radiuses $a_{\mathrm{p}}$. The solid curve and thin band denote the mean and standard deviation of 20 simulations, respectively. In each case, $\tilde{v}_{\mathrm{f}}$ increases significantly with the cluster formation in the early stage. Subsequently, $\tilde{v}_{\mathrm{f}}$ decreases with time because of the head shrinkage. The standard deviation is negligibly small after the head-tail structure is formed. This implies that the identical structure is formed independently of the initial condition.

To verify the particle movement in the head-tail structure, we focus on the $x$ component of the particle velocity. In Figure 7a, the speed of the white particle is slower compared to the front speed $v_{\mathrm{f}}$, whereas that of the gray particle is faster compared to $v_{\mathrm{f}}$. The particle speed in the front part of the granular flow (except the upper part of the head) exceeds $v_{\mathrm{f}}$, whereas that at the rear part (i.e., the tail) is smaller than $v_{\mathrm{f}}$. Therefore, the particle moves from the upper part of the head to the tail. These particle movements lead to the head shrinkage.

\section{Discussion}

\subsection{Definition of the Head in the $x-y$ Plane}

As mentioned in Sections 3.1 and 3.2, the front speed of the granular flow relates to the head size. The multihead structure is formed from the straight shape in the $x-y$ plane, which is parallel to the slope. Hereinafter, we define the head based on the particle number density to discuss the temporal variation of the head size and the size distribution of the head.

Our idea is to detect the particle with the relatively high particle number density from the flow pattern, as shown in Figure 8. As a simple index, we count two different types of numbers around the $i$ th particle, $n_{i}$ and $n_{i}^{\prime}$ as follows,

$$
\begin{aligned}
& n_{i}=\sum_{r_{i j}<4 a_{\mathrm{p}}}^{N} 1, \\
& n_{i}^{\prime}=\sum_{r_{i j}<4 a_{\mathrm{p}}}^{N} n_{j},
\end{aligned}
$$

where $r_{i j}$ is the interparticle distance between the $i$ th and $j$ th particles. Here, $n$ in Equation (11a) is the number of particles around the $i$ th particle, whereas $n$ indicates the number of indirect neighbors of $n$. In other words, $n^{\prime}$ yields the quasi-local particle number density.

According to the value of $n$ or $n$, the particle constituting the head is detected, as shown in Figure $8 \mathrm{a}, \mathrm{b}$. The procedure for calculating $n$ (or $n$ ) is described below. The value of $n$ depends on the aggregation size (i.e., the head). Therefore, we set the criterion for judging the constituent particle of the head using the maximum value $n_{\max }$ calculated for each aggregated part. If the $i$ th particle satisfies $n_{i} / n_{\max }>1 / 4$, this particle is assumed to be the constituent of the head. We apply the same procedure to $n$ '. Figure $8 \mathrm{a}, \mathrm{b}$ shows the head defined according to $n$ and $n^{\prime}$, respectively. The blue particle satisfies the criterion above; that is, it is a constituent of the head. Compared to the criteria for $n$ and $n^{\prime}$, the difference is obvious. The head extends backward along the outside edge of the granular flow in the case of $n$ (Figure 8a), whereas the head is located at the front of the granular flow in the case of $n^{\prime}$ (Figure $8 b$ ). In this study, we adopt the criterion with $n^{\prime}$ as the definition of the head.

To measure the head size, we select four targets from the constituent particles of the head, as shown in Figure 8c. Two green particles are selected as the outmost positions in the $y$ direction, and the others are selected as the forefronts of the outer and inner positions in the $x$ direction. Using these particles, the width and layer thickness of the head, $w_{\mathrm{h}}$ and $l_{\mathrm{h}}$, respectively, are measured (Figure $8 \mathrm{c}$ ). The calculations for the temporal variation in the head size and the head size distribution are explained in Section 4.2. 

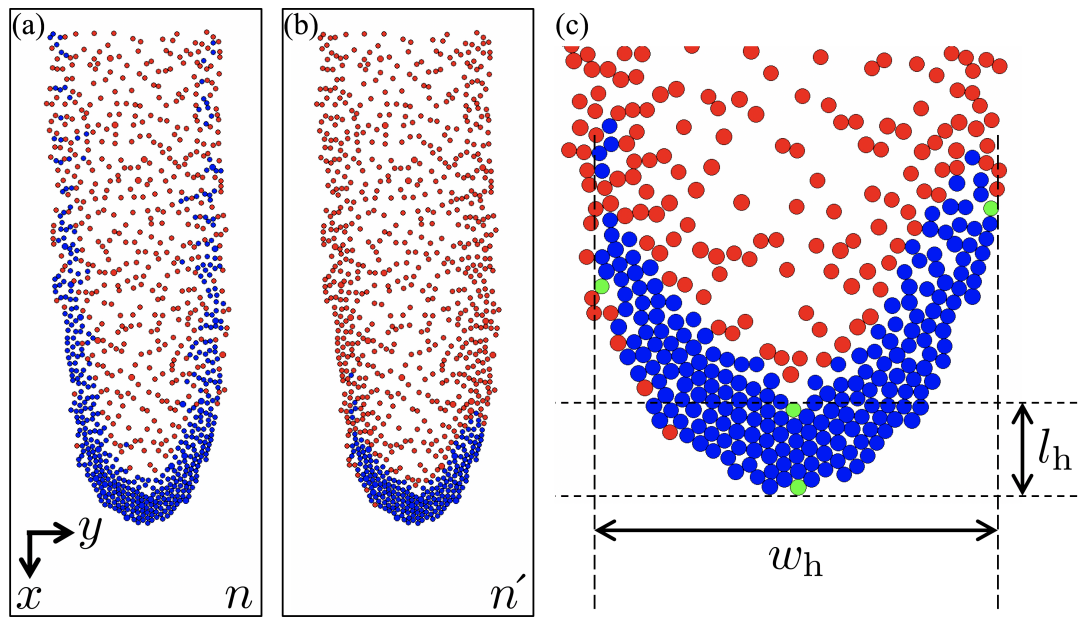

Figure 8. Examples of the head in the $x-y$ plane defined according to Equation (11): (a) $n$, (b), and (c) $n$. The blue and green particles constitute a head at the front of the granular flow, in which four green particles are selected as targets to measure the width $w_{\mathrm{h}}$ and layer thickness $l_{\mathrm{h}}$ of the head.

\subsection{Characteristics of Head Size in the $x-y$ Plane}

Based on the quasi-local particle number density $n$ in Equation (11b), we study the temporal variation of the head size from the granular flow in the circular shape (see Section 3.1). Because the simulation time is different for each particle radius $a_{\mathrm{p}}$, the width and layer thickness of the head, $w_{\mathrm{h}}$ and $l_{\mathrm{h}}$, respectively, are measured at different time intervals depending on $a_{\mathrm{p}}: \Delta t=1,0.5$, and $0.25 \mathrm{~ms}\left(a_{\mathrm{p}}=1,2.5\right.$, and $\left.5 \mathrm{~mm}\right)$. Therefore, the number of data is fixed as 20 in each simulation. Figure 9a shows the time evolutions of the number of particles constituting the head defined by the criterion $n^{\prime}$ in Equation (11b). The point and error bar denote the mean and standard deviation of 20 simulations, respectively. In the early stage, the head consists of almost half of the total number of particles $(N=2000)$, but the number of constituent particles rapidly decreases with time. Figure $9 \mathrm{~b}$ shows the time evolutions of the normalized width and thickness of the head, $\tilde{w}_{\mathrm{h}}(\tilde{t})$ and $\tilde{l}_{\mathrm{h}}(\tilde{t})$, which are denoted by the open and filled points, respectively. The normalized width and thickness decrease with time, leading to the shrinking of the head. Also, these decreasing trends of $\tilde{w}_{\mathrm{h}}$ and $\tilde{l}_{\mathrm{h}}$ are very similar to that of the number of particles constituting the head. To evaluate the relationship between the head size and the frontal speed, as shown in Figures 4 and 5a, we compare the number of particles constituting the head with the normalized frontal speed $\tilde{v}_{\mathrm{f}}$ (Figure 9c). The frontal speed of the granular flow becomes slower with the decrease in the number of particles constituting the head. Therefore, we find a good positive relation between the head size and frontal speed. 
(a)

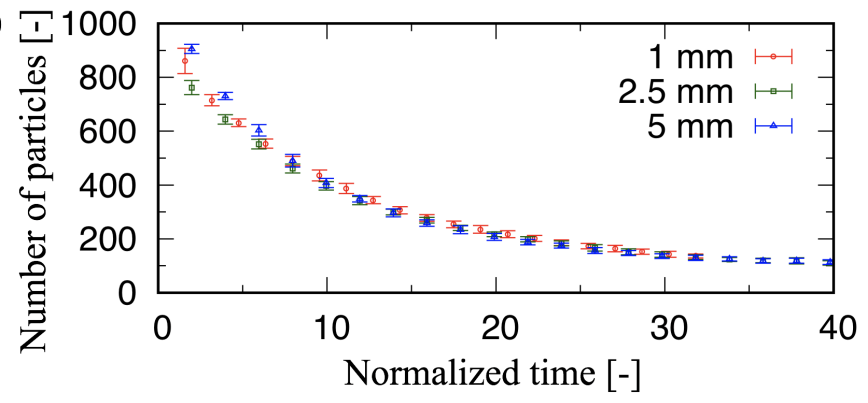

(b)

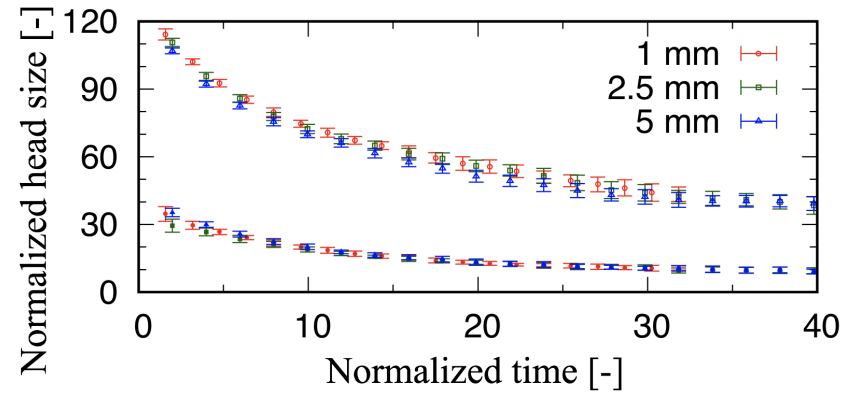

(c)

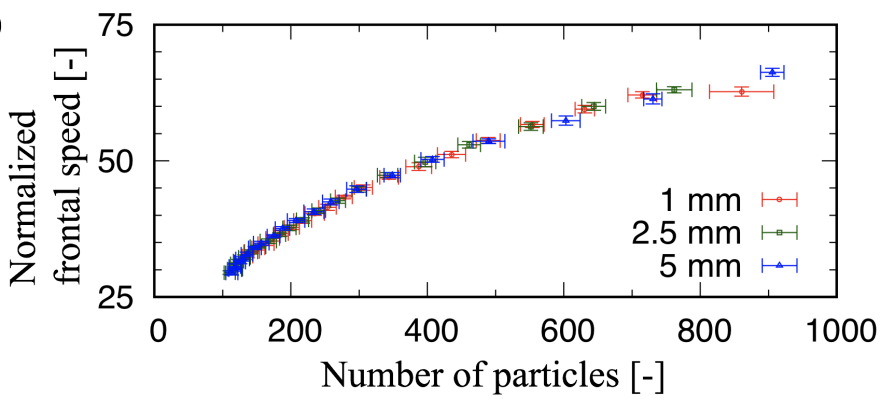

Figure 9. Time evolutions of head size defined by $n^{\prime}$ in Equation (11b) in the circular setup. The mean and standard deviation of 20 simulations are denoted by a point and an error bar, respectively. (a) Number of particles constituting the head (blue and green particles in Figure 8c). (b) Width and thickness of head normalized by particle radius, $\tilde{w}_{\mathrm{h}}$ and $\tilde{l}_{\mathrm{h}}$. The open and filled points denote $\tilde{w}_{\mathrm{h}}$ and $\tilde{l}_{h}$, respectively. (c) Relationship between the number of particles constituting the head and normalized frontal speed $\tilde{v}_{\mathrm{f}}$, as shown in Figure $5 \mathrm{c}$.

Next, we focus on the characteristics of the head shape in the circular and rectangular shapes. Figure 10a shows the relationship between $\tilde{w}_{\mathrm{h}}$ and $\tilde{l}_{\mathrm{h}}$. The point and error bar denote the mean and standard deviation of 20 simulations, respectively. During the head shrinkage, the ratio between width and layer thickness is maintained $\left(\tilde{w}_{\mathrm{h}} / \tilde{l}_{\mathrm{h}}=4\right)$, except during the early stage. We find that the similarity law for the head shape holds in the low-density granular flow. Moreover, we verify the head size distribution on the multihead structure of the rectangular shape (see Section 3.2). In this analysis, the head size is measured at the end of the simulations, where the head elongates downward, as shown in Figure $6 c$. In Figure $6 c$, the blue particles denote the constituent of the head, and multiple heads are detected. The measurement of the layer thickness $l_{\mathrm{h}}$ is difficult in this situation. Therefore, only the width $w_{\mathrm{h}}$ is measured as the head size. For the number of heads, the mean and standard deviation of 20 simulations at $a_{\mathrm{p}}=1,2.5$, and $5 \mathrm{~mm}$ are $(17.20,1.69),(13.95,1.94)$, and $(14.85,1.35)$, respectively. Figure $10 \mathrm{~b}$ shows the probability density of the normalized width $\tilde{w}_{\mathrm{h}}$. Here, $\tilde{w}_{\mathrm{h}}$ is distributed symmetrically and the distribution shape is independent of $a_{\mathrm{p}}$. This distribution is fitted well by the Gaussian distribution indicated by the dashed black line in Figure 10b. Although the fluctuation in $\tilde{w}_{\mathrm{h}}$ might be caused by the inhomogeneity of the initial condition, the flow instability at the moving front of the granular flow exhibits the characteristic wavelength. 
(a)

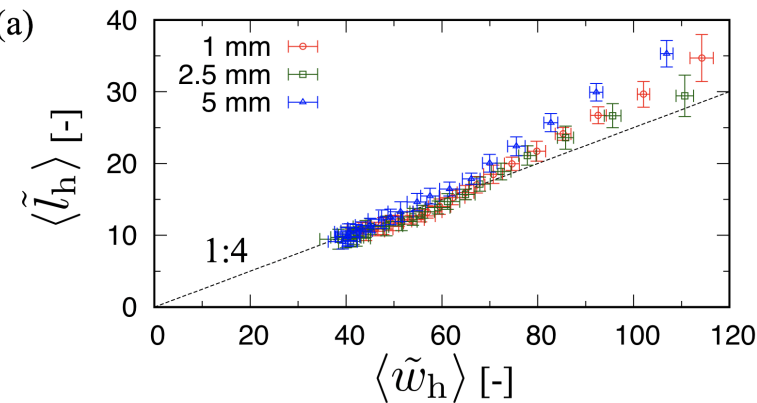

(b)

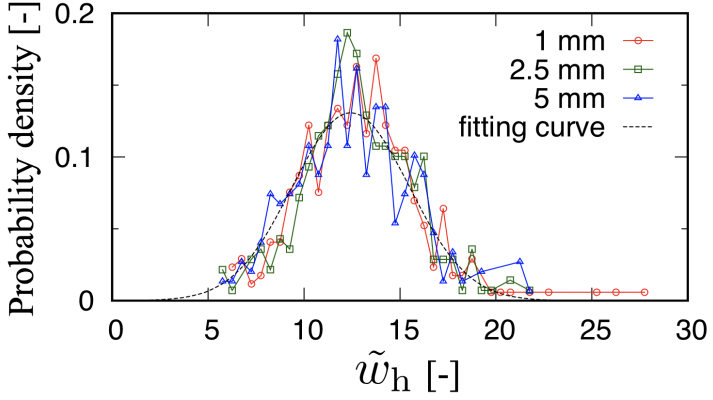

(c)

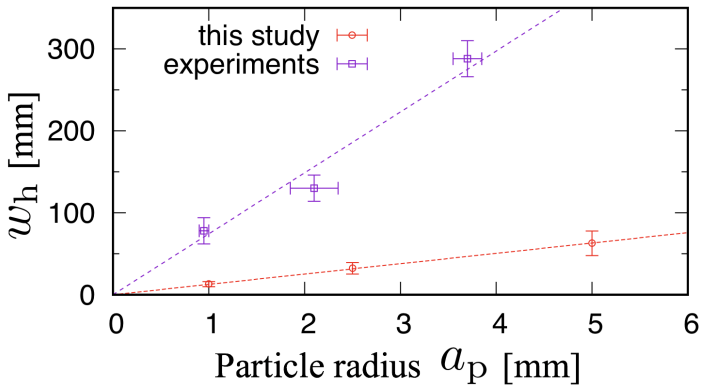

Figure 10. Variabilities in head size defined in Figure 8. Here, $\tilde{w}_{\mathrm{h}}$ and $\tilde{l}_{\mathrm{h}}$ are the width and layer thickness of the head normalized by the particle radius $a_{\mathrm{p}}$. (a) Relationship between $\tilde{w}_{\mathrm{h}}$ and $\tilde{l}_{\mathrm{h}}$ in the circular set-up. The mean and standard deviation of 20 simulations are denoted by a point and an error bar, respectively. The data are shown at a constant time interval depending on $a_{\mathrm{p}}: \Delta t=10^{-3}$, $5 \times 10^{-4}$, and $2.5 \times 10^{-4} \mathrm{~s}\left(a_{\mathrm{p}}=1,2.5\right.$, and $\left.5 \mathrm{~mm}\right)$. (b) Probability densities of $\tilde{w}_{\mathrm{h}}$ in the rectangular setup. Each line is calculated from the final states of 20 simulations. The dashed black line is a Gaussian distribution fitting the data of $a_{\mathrm{p}}=5 \mathrm{~mm}$. (c) Comparison of head width with previous experiments [23]. The mean and standard deviation of panel (b) are shown. The dashed lines are linear functions fitting the simulation and experimental data.

For the width of the head $w_{\mathrm{h}}$, we compare our result qualitatively with the previous experimental findings using polystyrene particles [23]. In the paper, flow instability resembling a wavy pattern was observed, and the frontal radius was estimated by fitting the circle against each head (see Table 1 in [23]). In this study, we assume that $w_{\mathrm{h}}$ corresponds to the diameter of the frontal shape (i.e., $2 \times$ radius) in the previous experiments. Figure 10c shows the relationship between particle radius $a_{\mathrm{p}}$ and $w_{\mathrm{h}}$ in this study and that in the previous experiments. The point and error bar denote the mean and standard deviation, respectively. In particular, the data in this study are calculated from the distribution shown in Figure 10b. Although the measured $w_{\mathrm{h}}$ in this study is much smaller than that in the experiments because of the small number of particles in the two-dimensional planes, each set of data fits the linear function well (dashed line in Figure 10c). We find that $w_{\mathrm{h}}$ is proportional to $a_{\mathrm{p}}$ in both cases.

\subsection{Frontal Angle in the $x-z$ Plane}

As mentioned in Section 3.3, the low-density granular flow is simulated in the $x-z$ plane and the head-tail structure is formed. The shape of the head was observed from the side view of the granular flow in previous experiments with ping pong balls or polystyrene particles $[20,25,32]$. Notably, the 
frontal angle of the head generally exhibits a high elevation angle to the slope and then becomes a constant angle $\left(60^{\circ}\right)$ according to the kinematic theory (see in [32]). Therefore, we measure the frontal angle of the head from the flow pattern, as shown in Figure $7 b, c$, to check if the result agrees with this fact.

The definition of frontal angle $\phi_{\mathrm{f}}$ is based on the coordinate of the particle located on the surface of the head. Figure 11a shows the typical shape of the head and the corresponding $\phi_{\mathrm{f}}$. To measure $\phi_{\mathrm{f}}$, we first select the forefront particle of the granular flow in the $x$ direction, namely, the green particle in Figure 11a. Next, we search the constituent of the head surface around the green particle, as shown in Figure 11b, where the search range spans the three particle radii and the search angle against the slope is $\varphi=0^{\circ}-180^{\circ}$. By repeating this search, the red particles in Figure 11 a are detected. A fitting line is obtained from the coordinates of the green and red particles using the least-squares method. Subsequently, $\phi_{\mathrm{f}}$ is estimated. It is noteworthy that the blue particles below the green particle are excluded from the estimation.
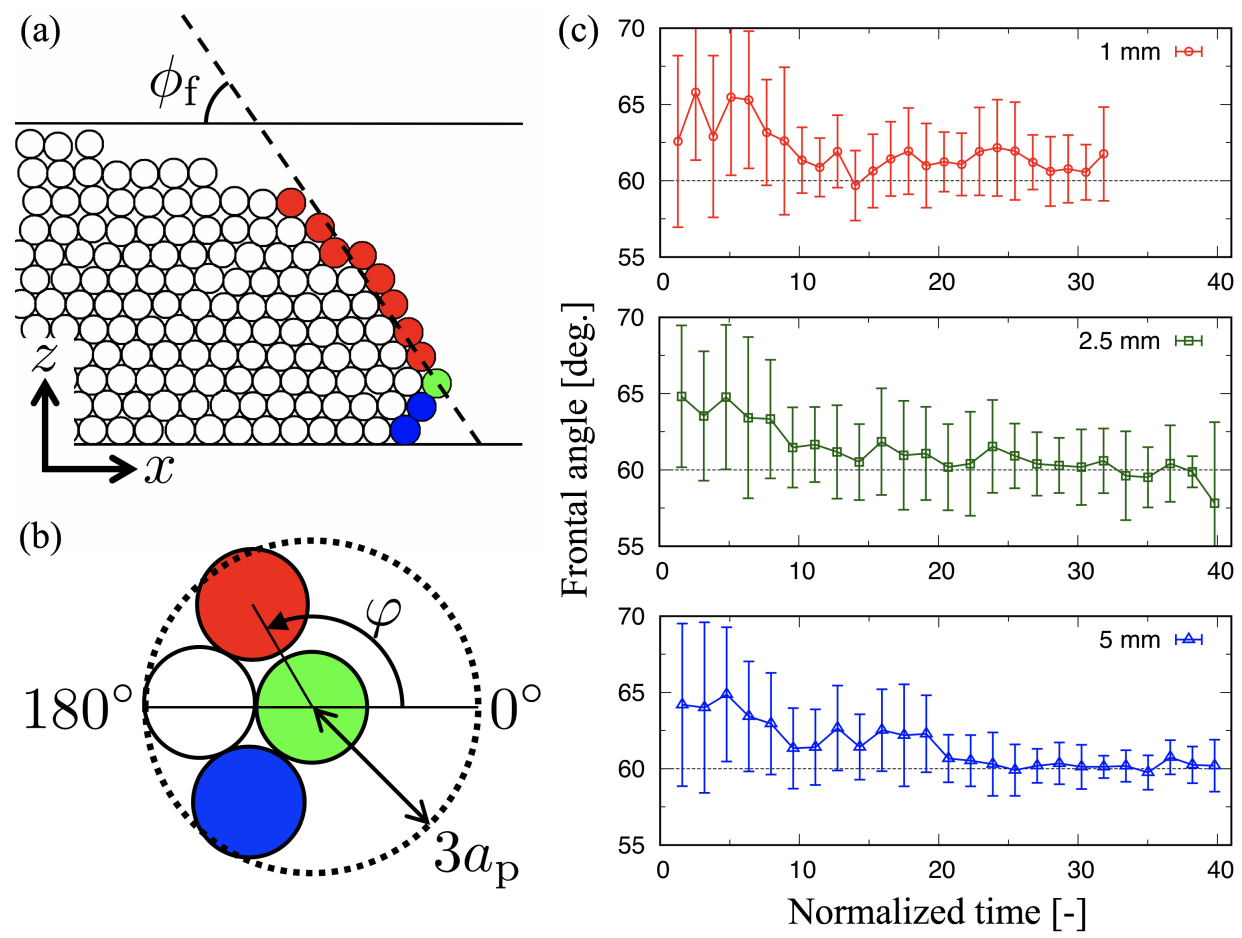

Figure 11. Definition and temporal variations of frontal angle $\phi_{\mathrm{f}}$ in the $x-z$ plane (triangular set-up). $(\mathbf{a}, \mathbf{b})$ The green particle is at the forefront of the granular flow, and the neighbor particle is selected within $3 a_{\mathrm{p}}$ and $\varphi=0^{\circ}-180^{\circ}$. The red particle is selected in a similar way, and the green particle contributes to $\phi_{\mathrm{f}}$, whereas the blue particle is excludable. The dashed black line is drawn using the least-squares method against the coordinates of the red and green particles. (c) The mean and standard deviation of 20 simulations are denoted by a point and error bar, respectively.

Figure 11c shows the temporal variation of the frontal angle $\phi_{\mathrm{f}}$ at each particle radius $a_{\mathrm{p}}$ in the triangular shape. The mean and standard deviation of 20 simulations are denoted by the point and error bar, respectively. In the initial condition, as shown in Figure $7 \mathrm{a}, \phi_{\mathrm{f}}$ is estimated as approximately $90^{\circ}$. Subsequently, $\phi_{\mathrm{f}}$ approaches $60^{\circ}$ from the larger angle as time progresses, and the standard deviation of $\phi_{\mathrm{p}}$ decreases with time in all cases. This convergence angle of $60^{\circ}$ is consistent with the frontal angle reported in previous studies [32]. However, $\phi_{\mathrm{f}}=60^{\circ}$ in this study results in the crystallization of particles in the two-dimensional simulation (Figure $7 \mathrm{~b}, \mathrm{c}$ ), although we use particles with a polydispersity of $\pm 5 \%$ to avoid this phenomenon. Even though a three-dimensional simulation is performed, such a crystallization is to be expected. 


\section{Conclusions}

In this study, three types of pattern formations for a low-density granular flow were reproduced qualitatively using the single particle model: a crescent-shaped head, a wavy pattern due to flow instability, and a head-tail structure. This model considered the gravity, repulsive force, and drag force due to the fluid as forces acting on the particle, thus providing the minimum number of factors to simplify this natural but complicated phenomenon. The frontal speed of the head decreased with time and was related positively to the head shrinkage (or number of constituent particles). Moreover, granular vortex convection was formed at the head in the $x-y$ plane parallel to the slope, whereas the particle moved from the upper and rear parts of the head to the tail in the $x-z$ plane perpendicular to the slope. According to the quasi-local particle number density, the width and layer thickness of the head were defined as the characteristic head size. The ratio (width/layer thickness) was maintained $(\approx 4)$ while the head shrunk, and the size distribution of the width obeyed the Gaussian distribution. For a comparison with previous experiments regarding the low-density granular flow, we found a linear relationship between the head width and particle radius. Additionally, the frontal angle of the head converged on $60^{\circ}$. This model is not realistic yet. However, we believe we pioneered a particle-based approach to elucidate the dynamics of low-density granular flow in this study.

Author Contributions: Conceptualization, H.N. (Hirofumi Niiya); methodology, H.N. (Hirofumi Niiya) and A.A.; validation, H.N. (Hiraku Nishimori); formal analysis, H.N. (Hirofumi Niiya); investigation, H.N. (Hirofumi Niiya); data curation, H.N. (Hirofumi Niiya); writing—original draft preparation, H.N. (Hirofumi Niiya); writing-review and editing, all authors; visualization, H.N. (Hirofumi Niiya); supervision, H.N. (Hirofumi Niiya); project administration, H.N. (Hirofumi Niiya); funding acquisition, H.N. (Hirofumi Niiya). All authors have read and agree to the published version of the manuscript.

Funding: This research was funded by JSPS KAKENHI grant numbers 11J07296, 14J03528, and 17K14353.

Acknowledgments: We thank Editage (www.editage.jp) for English language editing. We would like to thank 3 anonymous reviewers for carefully reading of our manuscript and providing constructive comments.

Conflicts of Interest: The authors declare no conflicts of interest. The funders had no role in the design of the study; in the collection, analyses, or interpretation of the data; in the writing of the manuscript; or in the decision to publish the results.

\section{References}

1. Sovilla, B.; McElwaine, J.N.; Louge, M.Y. The structure of powder snow avalanches. C. R. Physique 2015, 16, 97-104. [CrossRef]

2. Köhler, A.; McElwaine, J.; Sovilla, B.; Ash, M.; Brennan, P. The dynamics of surges in the 3 February 2015 avalanches in Vallée de la Sionne. J. Geophys. Res. Earth Surf. 2016, 121, 2192-2210. [CrossRef]

3. Iverson, R.M. The physics of debris flows. Rev. Geophys. 1997, 35, 245-296. [CrossRef]

4. Pouliquen, O.; Delour, J.; Savage, S.B. Fingering in granular flows. Nature 1997, 386, 816-817. [CrossRef]

5. Forterre, Y.; Pouliquen, O. Longitudinal vortices in granular flows. Phys. Rev. Lett. 2001, 86, 5886-5889. [CrossRef] [PubMed]

6. Tiefenbacher, F.; Kern, M.A. Experimental devices to determine snow avalanche basal friction and velocity profiles. Cold Reg. Sci. Technol. 2004, 38, 17-30. [CrossRef]

7. Gray, J.M.N.T.; Ancey, C. Segregation, recirculation and deposition of coarse particles near two-dimensional avalanche fronts. J. Fluid Mech. 2009, 629, 387-423. [CrossRef]

8. Gray, J.; Ancey, C. Multi-component particle-size segregation in shallow granular avalanches. J. Fluid Mech. 2011, 678, 535-588. [CrossRef]

9. Fischer, J.T.; Kaitna, R.; Heil, K.; Reiweger, I. The Heat of the Flow: Thermal Equilibrium in Gravitational Mass Flows. Geophys. Res. Lett. 2018, 45, 219-226. [CrossRef]

10. Thompson, P.A.; Grest, G.S. Granular flow: Friction and the dilatancy transition. Phys. Rev. Lett. 1991, 67, 1751-1754. [CrossRef]

11. Silbert, L.E.; Ertas, D.; Grest, G.S.; Halsey, T.C.; Levine, D.; Plimpton, S.J. Granular flow down an inclined plane: Bagnold scaling and rheology. Phys. Rev. E 2001, 64, 051302. [CrossRef] [PubMed] 
12. Savage, S.B.; Hutter, K. The motion of a finite mass of granular material down a rough incline. J. Fluid Mech. 1989, 199, 177-215. [CrossRef]

13. Forterre, Y.; Pouliquen, O. Stability analysis of rapid granular chute flows: formation of longitudinal vortices. J. Fluid Mech. 2002, 467, 361-387. [CrossRef]

14. Pitman, E.B.; Nichita, C.C.; Patra, A.; Bauer, A.; Sheridan, M.; Bursik, M. Computing granular avalanches and landslides. Phys. Fluids 2003, 15, 3638. [CrossRef]

15. Patra, A.K.; Bauer, A.C.; Nichita, C.C.; Pitman, E.B.; Sheridan, M.F.; Bursik, M.; Rupp, B.; Webber, A.; Stinton, A.J.; Namikawa, L.M.; et al. Parallel adaptive numerical simulation of dry avalanches over natural terrain. J. Volcanol. Geotherm. Res. 2005, 139, 1-21. [CrossRef]

16. Pailha, M.; Pouliquen, O. A two-phase flow description of the initiation of underwater granular avalanches. J. Fluid Mech. 2009, 633, 115-135. [CrossRef]

17. Beghin, P.; Hopfinger, E.J.; Britter, R.E. Gravitational convection from instantaneous sources on inclined boundaries. J. Fluid Mech. 1981, 107, 407-422. [CrossRef]

18. Beghin, P.; Olagne, X. Experimental and theoretical study of the dynamics of powder snow avalanches. Cold Reg. Sci. Technol. 1991, 19, 317-326. [CrossRef]

19. Nishimura, K.; Keller, S.; McElwaine, J.; Nohguchi, Y. Ping-pong ball avalanche at a ski jump. Granul. Matter 1998, 1, 51-56. [CrossRef]

20. McElwaine, J.; Nishimura, K. Ping-pong ball avalanche experiments. Ann. Glaciol. 2001, 32, 135-148. [CrossRef]

21. Tischer, M.; Bursik, M.I.; Pitman, E.B. Kinematics of Sand Avalanches Using Particle-Image Velocimetry. J. Sediment. Res. 2001, 71, 355-364. [CrossRef]

22. Turnbull, B.; McElwaine, J.N. Experiments on the non-Boussinesq flow of self-igniting suspension currents on a steep open slope. J. Geophys. Res. Earth Surf. 2008, 113. [CrossRef]

23. Nohguchi, Y.; Ozawa, H. On the vortex formation at the moving front of lightweight granular particles. Physica D 2009, 238, 20-26. [CrossRef]

24. Jackson, A.; Turnbull, B.; Munro, R. Scaling for lobe and cleft patterns in particle-laden gravity currents. Nonlin. Processes Geophys. 2013, 20, 121-130. [CrossRef]

25. Jackson, A.; Turnbull, B. Identification of particle-laden flow features from wavelet decomposition. Physica D 2017, 361, 12-27. [CrossRef]

26. Metzger, B.; Nicolas, M.; Guazzelli, E. Falling clouds of particles in viscous fluids. J. Fluid Mech. 2007, 580, 283-301. [CrossRef]

27. Issler, D. Modelling of snow entrainment and deposition in powder-snow avalanches. Ann. Glaciol. 1998, 26, 253-258. [CrossRef]

28. Naaim, M.; Gurer, I. Two-phase numerical model of powder avalanche theory and application. Nat. Hazards 1998, 17, 129-145. [CrossRef]

29. Hartel, C.; Meiburg, E.; Necker, F. Analysis and direct numerical simulation of the flow at a gravity-current head. Part 1. Flow topology and front speed for slip and no-slip boundaries. J. Fluid Mech. 2000, 418, 189-212. [CrossRef]

30. Hartel, C.; Carlsson, F.; Thunblom, M. Analysis and direct numerical simulation of the flow at a gravity-current head. Part 2. The lobe-and-cleft instability. J. Fluid Mech. 2000, 418, 213-229. [CrossRef]

31. Ancey, C. Powder snow avalanches: Approximation as non-Boussinesq clouds with a Richardson number-dependent entrainment function. J. Geophys. Res. 2004, 109. [CrossRef]

32. McElwaine, J.N. Rotational flow in gravity current heads. Phil. Trans. R. Soc. A 2005, 363, 1603-1623. [CrossRef] [PubMed]

33. Turnbull, B.; McElwaine, J.N. Potential flow models of suspension current air pressure. Ann. Glaciol. 2010, 51, 113-122. [CrossRef]

34. Espath, L.F.R.; Pinto, L.C.; Laizet, S.; Silvestrini, J.H. High-fidelity simulations of the lobe-and-cleft structures and the deposition map in particle-driven gravity currents. Phys. Fluids 2015, 27, 056604. [CrossRef]

35. Rotne, J.; Prager, S. Variational treatment of hydrodynamic interaction in polymers. J. Chem. Phys. 1969, 50, 4831. [CrossRef]

(C) 2020 by the authors. Licensee MDPI, Basel, Switzerland. This article is an open access article distributed under the terms and conditions of the Creative Commons Attribution (CC BY) license (http://creativecommons.org/licenses/by/4.0/). 